\title{
Associations of adiponectin with individual European ancestry in African Americans: the Jackson Heart Study
}

\section{Aurelian Bidulescu ${ }^{1 *}$, Shweta Choudhry ${ }^{2}$, Solomon K. Musani ${ }^{3}$, Sarah G. Buxbaum ${ }^{4}$, Jiankang Liu ${ }^{3}$, Charles N. Rotimi ${ }^{5}$, James G. Wilson ${ }^{3}$, Herman A. Taylor ${ }^{3}$ and Gary H. Gibbons ${ }^{6}$}

${ }^{1}$ Department of Epidemiology and Biostatistics, Indiana University School of Public Health - Bloomington, Bloomington, IN, USA

2 Department of Urology, University of California, San Francisco, CA, USA

3 Jackson Heart Study, University of Mississippi Medical Center, Jackson, MS, USA

${ }^{4}$ Department of Health Sciences, Jackson State University, Jackson, MS, USA

${ }^{5}$ National Human Genome Research Institute, National Institutes of Health, Bethesda, MD, USA

${ }^{6}$ National Heart Lung and Blood Institute, National Institutes of Health, Bethesda, MD, USA

\section{Edited by:}

Struan F. A. Grant, Children's Hospital of Philadelphia Research Institute, USA

\section{Reviewed by:}

Hui-Qi Qu, The University of Texas

School of Public Health, USA

Inga Reynisdóttir, Landspitali

University-Hospital, Iceland

Swapan K. Das, Wake Forest School

of Medicine, USA

\section{*Correspondence}

Aurelian Bidulescu, Department of Epidemiology and Biostatistics, Indiana University School of Public Health-Bloomington, $1025 \mathrm{E}$ 7th

Street, Bloomington, IN 47405, USA e-mail: abidules@indiana.edu

Background: Compared with European Americans, African Americans (AAs) exhibit lower levels of the cardio-metabolically protective adiponectin even after accounting for adiposity measures. Because few studies have examined in AA the association between adiponectin and genetic admixture, a dense panel of ancestry informative markers (AIMs) was used to estimate the individual proportions of European ancestry (PEA) for the AAs enrolled in a large community-based cohort, the Jackson Heart Study (JHS). We tested the hypothesis that plasma adiponectin and PEA are directly associated and assessed the interaction with a series of cardio-metabolic risk factors.

Methods: Plasma specimens from 1439 JHS participants were analyzed by ELISA for adiponectin levels. Using pseudo-ancestral population genotype data from the HapMap Consortium, PEA was estimated with a panel of up to 1447 genome-wide preselected AIMs by a maximum likelihood approach. Interaction assessment, stepwise linear and cubic multivariable-adjusted regression models were used to analyze the cross-sectional association between adiponectin and PEA.

Results: Among the study participants (62\% women; mean age $48 \pm 12$ years), the median (interquartile range) of PEA was 15.8 (9.3)\%. Body mass index (BMI) $(p=0.04)$ and insulin resistance $(p=0.0001)$ modified the association between adiponectin and PEA. Adiponectin was directly and linearly associated with PEA $(\beta=0.62 \pm 0.28, p=0.03)$ among non-obese $(n=673)$ and insulin sensitive participants $(n=1141 ; \beta=0.74 \pm 0.23$, $p=0.001$ ), but not among those obese or with insulin resistance. No threshold point effect was detected for non-obese participants.

Conclusions: In a large AA population, the individual proportion of European ancestry was linearly and directly associated with plasma adiponectin among non-obese and non insulin-resistant participants, pointing to the interaction of genetic and metabolic factors influencing adiponectin levels.

Keywords: cohort study, adiponectin, individual European ancestry, minorities, African Americans, obesity, insulin resistance

\section{INTRODUCTION}

Complex and common chronic diseases such as cardiovascular disease (CVD) which affect African Americans (AAs) disproportionally are likely caused by many genetic and environmental factors and their interactions (Go et al., 2014). Assessing associations between self-reported race/ethnicity with these diseases is often complicated due to heterogeneity within racial/ethnic groups (Burchard et al., 2003). The most abundant product of adipocytes, adiponectin is a global endophenotype for obesity and cardio-metabolic disease risk (Choi et al., 2004; Vettor et al., 2005; Wannamethee et al., 2007; Bidulescu et al., 2013a), and a potential mediator of the cardio-metabolic consequences of obesity (Ouchi et al., 2006). When compared to European Americans,
AAs exhibit lower levels of the cardio-metabolically protective adipokine named adiponectin even after accounting for adiposity measures (Patel et al., 2006; Bidulescu et al., 2013b). The reason for this ethnic difference is still unclear.

African Americans in the United States are variably admixed with African and European ancestry (Parra et al., 2001). Due to this heterogeneity, genetic admixture analysis offers a unique opportunity for studying the role of genetic factors within a single, admixed population, controlling for socioeconomic and behavioral factors as well as comorbidities (Pritchard et al., 2000). Nevertheless, few studies have examined in AAs the association between adiponectin and individual ancestry estimates, as estimated with ancestry informative markers (AIMs), genetic loci 
showing alleles with large frequency differences between populations (Wassel Fyr et al., 2007). In addition, these studies used relatively few AIMs and small AA sample sizes.

We thus employed a relatively dense panel of AIMs to estimate the individual proportions of European ancestry (PEA) for the AAs enrolled in a large community-based cohort, the Jackson Heart Study (JHS) (Smith et al., 2004; Xu and Jin, 2008), in order to assess the association of this proportion with serum levels of the adiponectin. The hypothesis tested was that serum adiponectin and PEA are directly associated. For interaction assessment purposes, we also queried if obesity, insulin resistance and other cardio-metabolic measures modify this association.

\section{MATERIALS AND METHODS STUDY POPULATION}

JHS is a single-site, prospective cohort study of the risk factors and causes of heart disease in adult AAs. A probability sample of 5301 AAs, aged 21-94 years, residing in a three county area surrounding the city of Jackson, MS, were recruited and examined at baseline (2000-2004) by certified technicians according to standardized protocols. Clinic visits and interviews occur approximately every 4 years. Annual follow-up interviews and cohort surveillance are ongoing. An overview of the JHS (Taylor, 2005) and details of the study design (Carpenter et al., 2004), recruitment protocol (Wyatt et al., 2003) and data collection methods (Carpenter et al., 2004) are published elsewhere.

The sample for this study included 1439 JHS participants $(62 \%$ women; mean age $48 \pm 12$ years) with available DNA through the Candidate-Gene Association Resource (CARe) Study (Musunuru et al., 2010) and that had serum adiponectin measured (Bidulescu et al., 2011). Written consent was obtained from each participant at the inception of the study, and the study was approved by the participating JHS institutions: Jackson State University, Tougaloo College and the University of Mississippi Medical Center. The study protocol for the ascertainment of the adiponectin samples was approved by the Morehouse School of Medicine Institutional Review Board.

\section{ADIPONECTIN MEASUREMENTS}

Venous blood samples were withdrawn from each subject at baseline examination after more than $8 \mathrm{~h}$ of fasting as described elsewhere (Carpenter et al., 2004). Vials of serum were stored at the JHS central repository in Minneapolis, $\mathrm{MN}$, at $-80^{\circ} \mathrm{C}$ until assayed. Adiponectin concentration was measured as total adiponectin by an ELISA system (R\&D Systems; Minneapolis, MN) (Bidulescu et al., 2011). The inter-assay coefficient of variation was $8.8 \%$. No biological degradation has been described using stored specimens, indicating a high validity for our measurements.

\section{PROPORTION OF EUROPEAN ANCESTRY}

In order to estimate ancestry we used a set of particularly informative DNA polymorphisms that have been termed AIMs due to their information content for distinguishing particular ancestral groups that correspond to continental populations. The proportion of European ancestry (as an estimate of the global proportion of individual European ancestry) was estimated for each individual using a panel of 1447 AIMs distributed across the genome. AIMs were selected using pseudo-ancestral population genotype data from the International HapMap Consortium to be informative for African vs. European ancestry (Smith et al., 2004; Xu and Jin, 2008). Ancestry proportions were estimated using a maximum likelihood approach in which the sum of the log-likelihood of individual ancestry from all the markers was maximized as a function of that person's ancestry, with a range between 0 and 1 (Chakraborty and Weiss, 1986; Long, 1991; Parra et al., 1998). If one considers an admixed population, K3, as the result from the genetic admixture of subjects from two ancestral populations, $\mathrm{K} 1$ and $\mathrm{K} 2$, then $s 1$ and (1-s1) will represent the ancestry proportion from population $\mathrm{K} 1$ and $\mathrm{K} 2$, separately. If one represents $G_{i}$ as the genotype for an admixed individual at the $i$ th locus, then for $n$ loci, likelihood can be defined as: $L(s 1)=\prod i=1$ to $n \operatorname{Pr}\left(G_{i}\right)$, where $\operatorname{Pr}$ is the actual proportion.

Instead of maximizing likelihood, it is computationally simple to maximize its natural logarithm: $\log _{e}[L(s 1)]=\sum i=1$ to $n$ $\log _{e}\left[\operatorname{Pr}\left(G_{i}\right)\right]$.

The MLE approach has been implemented in the program IAE3CI, which was kindly provided by Dr. Mark D. Shriver. The program requires the information of allele frequencies from each ancestral population and admixed subjects' genotyping data (Chakraborty et al., 1986; Hanis et al., 1986; Bonilla et al., 2004) We also compared our method with the algorithm for global ancestry estimation implemented in the other used programs. Tsai et al. (2005) evaluated the performances of three different methods for estimating global genetic ancestry: MLE, ADMIXMAP and STRUCTURE, through various simulated data sets and real data from Latino subjects participating in a genetic study of asthma. All three methods provided similar information on the accuracy of ancestral estimates. The Pearson's correlation coefficients, $r$, for ancestry estimates were $>0.99$ between MLE, ADMIXMAP, and STRUCTURE.

\section{CVD RISK FACTORS USED AS COVARIATES}

CVD risk factor information was ascertained during the JHS Exam 1 (2000-2004) through standard procedures and questionnaires. In all participants, the clinic visit included physical examinations, anthropometry, survey of medical history and current medication use and collection of blood and urine specimens for biological assessment. In-clinic standing height and weight were measured in lightweight examination clothing without shoes or constricting garments. We calculated body mass index (BMI) as weight in kilograms divided by height in meters squared $\left(\mathrm{kg} / \mathrm{m}^{2}\right)$. The average of two sitting blood pressure, measured at 1-min intervals after a 5-min silent rest, was used for analysis. Participants were considered current smokers if they smoked at the time of the baseline examination. Current alcohol use was defined as "yes" if they drank in the past 12 months. Lipid variables, fasting plasma glucose and fasting insulin were measured using standard laboratory techniques. Insulin resistance was calculated using the homeostasis model assessment for insulin resistance (HOMA-IR) (Matthews et al., 1985), and used as a continuous variable as well as a categorical variable. As a categorical variable, insulin resistance was defined as the upper 
quartile of the distribution as indicated by the fact that, considering the cardio-metabolic risk to establish the cut-off points for HOMA-IR, values between the 70th and the 75th percentile of HOMA-IR levels appears appropriate (Gayoso-Diz et al., 2013). Diabetes was defined as a fasting plasma glucose level of at least $7.0 \mathrm{mmol} / \mathrm{l}$ or if the subject was being treated with insulin or a hypoglycemic agent. Physical activity was assessed with a physical activity survey instrument administered by interview. The questionnaire assessed four different domains of physical activity (active living, work, home and garden, and sport and exercise indexes). A total score was composed as the sum of these domains (maximum of 24), with a higher score indicating a higher level of total physical activity (Dubbert et al., 2005). Obesity was defined by a $B M I \geq 30 \mathrm{~kg} / \mathrm{m}^{2}$. C-reactive protein (CRP) was measured in duplicate by the immunoturbidimetric CRP-Latex assay from Kamiya Biomedical Company using a Hitachi 911 analyzer, according to the manufacturer's high-sensitivity protocol. The inter-assay coefficients of variation on control samples repeated in each assay were $4.5 \%$ at a CRP concentration of $0.45 \mathrm{mg} / \mathrm{L}$ and $4.4 \%$ at $1.56 \mathrm{mg} / \mathrm{L}$.

\section{STATISTICAL ANALYSIS}

A cross-sectional analysis was performed. For each individual CVD risk factor, the Student unpaired $t$-test or the chi-square test was implemented in order to assess the significance of the difference between participants by sex.

Serum adiponectin levels were log-transformed in order to normalize their distribution and to meet assumptions about normality. Age- and sex-adjusted Pearson correlation coefficients between adiponectin, proportion of European ancestry and the main study covariates were calculated. Assessment of the interaction by sex, BMI, waist circumference, and insulin resistance were conducted using a log-likelihood testing. The effect measure modification potential of obesity and insulin resistance was assessed using interaction terms in the fully adjusted models. Multivariable linear regression models with a stepwise forward selection procedure were constructed with adiponectin as the dependent variable and PEA as the main independent variable. To confirm our findings, we have run a sensitivity analysis among participants without diabetes. For all analyses, the statistical significance was set at $P \leq 0.05$ for main and interactive effect.

We also investigated the structure of the relationship between log-transformed serum adiponectin levels with proportion of European ancestry using cubic regression splines. In a multivariable-adjusted generalized additive model, we compared the deviance between models with and without proportion of ancestry. A non-linear relation was determined by a significant deviance and estimated degrees of freedom greater than 3 .

All computations were performed using the SAS software version 9.2 (SAS ${ }^{\circledR}$ Institute Inc., Cary, North Carolina).

\section{RESULTS}

Our study sample comprised 897 women and 542 men. The average age was $48 \pm 12$ years. The serum adiponectin levels were statistically different between sexes: $5.3 \pm 3.7 \mu \mathrm{g} / \mathrm{mL}$ in women and $3.8 \pm 2.7 \mu \mathrm{g} / \mathrm{mL}$ in men. Among our study participants, the median (interquartile range) of PEA was 15.8 (9.3)\%. There were statistically significant differences between sexes for adiponectin, BMI, waist circumference, triglycerides, HDL-cholesterol, fasting plasma glucose, HOMA-IR, type 2 diabetes, and CRP (Table 1) Among our study participants, 766 were obese, 209 were classified as having type 2 diabetes and 298 as insulin resistant participants (by HOMA-IR); 249 participants do not have insulin level measured, and thus did not have HOMA-IR estimated.

\section{CORRELATIONS BETWEEN ADIPONECTIN PROPORTION OF EUROPEAN ANCESTRY AND OTHER VARIABLES}

Age- and sex-adjusted correlates of log-transformed adiponectin with the proportion of European ancestry and the CVD risk factors considered in our study are presented in Table 2. Adiponectin was significantly associated with the majority of covariates with the exception of systolic blood pressure, physical activity, and proportion of European ancestry (Table 2). Proportion of European ancestry was significantly associated with adiponectin and waist circumference, but not with HOMA-IR or BMI (Table 2). Thus, bivariate analyses among all our study participants revealed a statistically significant but weak correlation between PEA and adiponectin.

\section{MULTIVARIABLE-ADJUSTED LINEAR REGRESSION MODELS OF ADIPONECTIN AND PROPORTION OF EUROPEAN ANCESTRY}

Body mass index $(p=0.04)$ and insulin resistance $(p=0.0001)$ were effect modifiers of the association PEA-adiponectin. On

Table 1 | Descriptive characteristics (mean \pm standard deviation, or percentage) of study participants $(N=1439)$ stratified by sex.

\begin{tabular}{|c|c|c|c|}
\hline & $\begin{array}{l}\text { Women } \\
(N=897)\end{array}$ & $\begin{array}{c}\text { Men } \\
(N=542)\end{array}$ & $\begin{array}{l}\text { Whole } \\
\text { sample }\end{array}$ \\
\hline Age (years) & $47 \pm 12$ & $49 \pm 11$ & $48 \pm 12$ \\
\hline Adiponectin $(\mu \mathrm{g} / \mathrm{mL})$ & $5.3 \pm 3.7$ & $3.8 \pm 2.7^{*}$ & $4.7 \pm 3.4$ \\
\hline PEA & $17.0 \pm 8.9$ & $17.8 \pm 8.1$ & $17.3 \pm 8.1$ \\
\hline $\mathrm{BMI}\left(\mathrm{kg} / \mathrm{m}^{2} ; n=1437^{* *}\right)$ & $33.0 \pm 7.4$ & $29.5 \pm 5.7^{*}$ & $31.7 \pm 7.0$ \\
\hline WC $(\mathrm{cm} ; n=1438)$ & $99.3 \pm 16.5$ & $99.0 \pm 14.2^{*}$ & $99.2 \pm 15.7$ \\
\hline $\mathrm{TG}(\mathrm{mg} / \mathrm{dL} ; n=1343)$ & $97.0 \pm 70.0$ & $113.5 \pm 118.5^{*}$ & $103.3 \pm 91.7$ \\
\hline HDL-C (mg/dL; 1344) & $53.6 \pm 13.8$ & $45.5 \pm 11.5^{*}$ & $50.5 \pm 13.6$ \\
\hline $\mathrm{FG}(\mathrm{mg} / \mathrm{dL} ; n=1344)$ & $95.3 \pm 27.5$ & $96.7 \pm 30.1^{*}$ & $95.8 \pm 28.5$ \\
\hline HOMA-IR $(n=1190)$ & $3.7 \pm 2.5$ & $3.2 \pm 2.0^{*}$ & $3.5 \pm 2.3$ \\
\hline Type 2 diabetes $(\% ; n=212$ ) & 16.3 & $12.1^{*}$ & 14.7 \\
\hline $\mathrm{SBP}(\mathrm{mm} \mathrm{Hg} ; n=1437)$ & $121.5 \pm 17.4$ & $123.0 \pm 16.2$ & $122.1 \pm 17.0$ \\
\hline $\mathrm{DBP}(\mathrm{mm} \mathrm{Hg} ; n=1437)$ & $77.7 \pm 10.0$ & $81.1 \pm 10.5$ & $79.0 \pm 10.3$ \\
\hline PA & $8.8 \pm 2.4$ & $9.2 \pm 2.4$ & $8.9 \pm 2.4$ \\
\hline Alc. Drink. (\% yes) & 50 & 66 & 56 \\
\hline $\mathrm{CRP}(\mathrm{mg} / \mathrm{dL})$ & $6.1 \pm 7.9$ & $3.0 \pm 5.5^{*}$ & $4.9 \pm 7.2$ \\
\hline Education ( $\%$ > High School) & 38 & 36 & 37 \\
\hline
\end{tabular}

PEA indicates proportion of European ancestry; BMI, body mass index; WC, waist circumference; TG, triglycerides; HDL-C, high-density lipoprotein cholesterol; FG, fasting plasma glucose; HOMA-IR, insulin resistance by homeostasis assessment model HOMA-IR; SBP, systolic blood pressure; DBP, diastolic blood pressure; $P A$, total physical activity score; Alc. Drink., Alcohol drinking in the past year; CRP, C-reactive protein.

${ }^{*}$ Statistical significant difference (t-test) by sex.

** Characteristics/parameters with less than the maximum number of 1439 participants are indicated in brackets (as the $n$ number). 
Table 2 | Age- and sex-adjusted Pearson correlation coefficients between adiponectin, proportion of European ancestry and covariates $(N=1439)$.

\begin{tabular}{lccccc}
\hline & \multicolumn{2}{c}{ Adiponectin* } & & Proportion of European ancestry \\
\cline { 2 - 3 } \cline { 5 - 6 } & Coefficient & $\boldsymbol{p}$-value & & Coefficient & p-value \\
\hline Adiponectin* & - & - & & 0.06 & 0.03 \\
PEA & 0.06 & 0.03 & & - & - \\
BMI (kg/m²) & -0.19 & $<0.0001$ & & -0.05 & 0.11 \\
WC (cm) & -0.25 & $<0.0001$ & & -0.05 & $<0.0001$ \\
TG & -0.21 & $<0.0001$ & & 0.05 & 0.11 \\
HDL-C & 0.29 & $<0.0001$ & & -0.02 & 0.52 \\
FG & -0.22 & $<0.0001$ & & -0.05 & 0.09 \\
HOMA-IR & -0.33 & $<0.0001$ & & -0.03 & 0.38 \\
SBP & -0.03 & 0.31 & & -0.04 & 0.15 \\
DBP & -0.04 & 0.19 & & -0.03 & 0.36 \\
PA & -0.01 & 0.76 & & -0.06 & 0.04 \\
CRP & -0.14 & $<0.0001$ & -0.01 & 0.66 \\
\hline
\end{tabular}

PEA indicates proportion of European ancestry; BMI, body mass index; WC, waist circumference; $p$, $p$-values; TG, triglycerides; $H D L$, high density lipoprotein cholesterol; FPG, fasting plasma glucose; HOMA-IR, insulin resistance (homeostasis model assessment); SBP, systolic blood pressure; DBP, diastolic blood pressure; PA, total physical activity score; CRP, C-reactive protein.

* Logarithmically-transformed values.

the contrary, $\operatorname{sex}(p=0.64)$ and waist circumference $(p=0.83)$ were not found to be effect modifiers. Among non-obese individuals $(n=673)$, adiponectin was directly associated with PEA ( $\beta=0.62 \pm 0.28, p=0.03)$, after adjustment for sex, waist circumference, systolic blood pressure, HOMA-IR, HDL-cholesterol and physical activity, as indicated by the stepwise procedure (Table 3).

Among participants without insulin resistance $(n=1141)$, adiponectin was also directly associated with PEA $(\beta=0.74 \pm$ 0.23, $p=0.001$ ), after adjustment for age, sex, BMI, waist circumference, HDL-cholesterol and CRP, as indicated by the stepwise procedure (Table 3 ). In our sensitivity analyses, among participants without diabetes $(n=1106)$, there was a similar direct association between adiponectin and PEA $(\beta=$ $0.52 \pm 0.22, p=0.02$ ).

\section{LINEAR RELATIONSHIP BETWEEN ADIPONECTIN AND PROPORTION OF EUROPEAN ANCESTRY}

Figure 1 shows plots relating PEA to adiponectin after multivariable adjustment and stratification by obesity status (Figures 1A,B) and by insulin resistance status (Figures 1C,D). Test of deviation from linearity showed that log adiponectin was linearly and significantly $(p=0.021)$ related to PEA in the non-obese participants but not in the obese group (Figure 1B vs. Figure 1A). It also showed that adiponectin was non-linearly and borderline significantly $(p=0.091)$ related to PEA in a group of insulin resistant participants (results not shown), but after fitting a cubic regression smoother with greater than three degrees of freedom, it was obvious that the relation was linear but not significant ( $p=0.418$; Figure 1C). Adiponectin was on the other hand linearly and highly significantly $(p=0.0094)$ related
Table 3 | Linear multivariable association between adiponectin and proportion of European ancestry.

\begin{tabular}{lccccc}
\hline By obesity status & \multicolumn{2}{c}{ Non-obese $(\boldsymbol{N}=\mathbf{6 7 3})^{*}$} & & \multicolumn{2}{c}{ Obese $(\boldsymbol{N}=\mathbf{7 6 6})^{*}$} \\
\cline { 2 - 3 } \cline { 5 - 6 } & $\beta$-coefficient & $\boldsymbol{p}$-value & & $\beta$-coefficient & $\boldsymbol{p}$-value \\
\hline PEA & 0.62 & 0.03 & & - & 0.81 \\
Age & - & 0.61 & & 0.005 & 0.04 \\
Sex & -0.28 & $<0.0001$ & & -0.32 & $<0.0001$ \\
WC & -0.01 & 0.01 & & - & 0.31 \\
TG & - & 0.77 & & - & 0.99 \\
HDL & 0.01 & $<0.0001$ & & 0.01 & $<0.0001$ \\
HOMA-IR & -0.09 & $<0.0001$ & & -0.06 & $<0.0001$ \\
SBP & 0.003 & 0.06 & & - & 0.10 \\
DBP & - & 0.38 & & - & 0.73 \\
PA & -0.02 & 0.06 & & - & 0.98 \\
Alc. Drink. & - & 0.33 & & - & 0.91 \\
CRP & - & 0.29 & & -0.08 & 0.01 \\
Education & - & 0.22 & & - & 0.56 \\
\hline
\end{tabular}

\begin{tabular}{lccccc}
\hline By insulin resist. status & \multicolumn{2}{c}{ Without IR $(\boldsymbol{N}=\mathbf{1 1 4 1})^{* *}$} & & \multicolumn{2}{c}{ With IR $(\boldsymbol{N}=\mathbf{2 9 8})^{* * *}$} \\
\cline { 2 - 3 } \cline { 5 - 6 } & $\beta$-coefficient & $\boldsymbol{p}$-value & & $\beta$-coefficient & $\boldsymbol{p}$-value \\
\hline PEA & 0.74 & 0.001 & & - & 0.41 \\
Age & 0.005 & 0.005 & & 0.006 & 0.06 \\
Sex & -0.25 & $<0.0001$ & & -0.24 & 0.001 \\
BMI & 0.01 & 0.003 & & - & 0.41 \\
WC & -0.01 & $<0.0001$ & & - & 0.28 \\
TG & - & 0.30 & & - & 0.90 \\
HDL & 0.01 & $<0.0001$ & & 0.01 & 0.02 \\
SBP & - & 0.16 & & - & 0.18 \\
DBP & - & 0.19 & & - & 0.93 \\
PA & - & 0.10 & & - & 0.61 \\
Alc. Drink. & - & 0.41 & & - & 0.70 \\
CRP & -0.08 & 0.01 & & - & 0.37 \\
Education & - & 0.83 & & 0.006 & 0.06 \\
\hline
\end{tabular}

PEA indicates proportion of European ancestry; BMI, body mass index; WC, waist circumference; TG, triglycerides; HDL, high density lipoprotein cholesterol; HOMA-IR, insulin resistance (homeostasis model assessment); SBP, systolic blood pressure; $D B P$, diastolic blood pressure; $P A$, total physical activity score; Alc. Drink., Alcohol drinking in the past year; CRP, C-reactive protein.

${ }^{*} R^{2}$ for the whole model $=0.22$.

${ }^{* *} R^{2}$ for the whole model $=0.10$.

${ }^{* * *} R^{2}$ for the whole model $=0.20$.

to PEA among non-insulin resistant participants (Figure 1D). Overall the structure of relation between adiponectin to PEA seems to be linear, especially among non-obese participants.

\section{DISCUSSION}

\section{PRINCIPAL FINDINGS}

In a large community-based AA sample, the individual proportion of European ancestry was directly associated with serum adiponectin among non-obese and among insulin sensitive participants. There was not an association among obese participants or among those with insulin resistance. Across the entire distribution of adiponectin, there was a linear and statistically significant direct association between adiponectin and PEA among non-obese participants. 


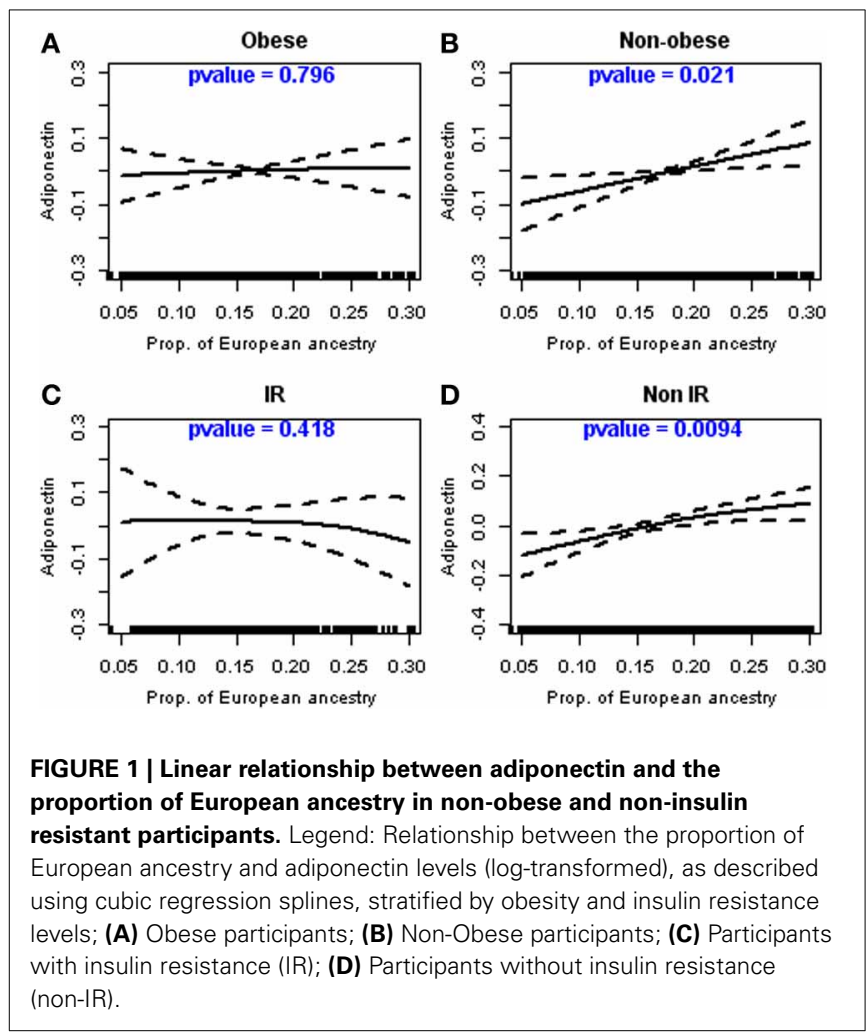

\section{IN THE CONTEXT OF PREVIOUS LITERATURE}

Few previous studies used estimates of individual ancestry to assess its association with CVD or other phenotypes (Wassel et al., 2009; Allison et al., 2010). A multivariable analysis in the Health $\mathrm{ABC}$ study showed a strong association between higher European ancestry and adiponectin levels (Wassel Fyr et al., 2007). The mean (standard deviation) of European ancestry among the 1241 AAs enrolled in that study was 22.3 (15.9)\%, thus slightly higher compared to our investigation (Wassel Fyr et al., 2007). Estimates of European ancestry in our cohort were more consistent with other studies among AAs. For example, Parra and colleagues observed around 20\% European ancestry for AA individuals from Pittsburgh, PA and around $12 \%$ in Charleston, SC (Parra et al., 1998).

African Americans in the United States are a heterogeneous group, variably admixed with African and European ancestry (Parra et al., 2001; Campbell et al., 2005). Because geneticassociation studies are often performed in population samples of unrelated individuals to identify susceptibility loci for complex human traits, if subjects are sampled from two or more subpopulations for which the frequencies of marker alleles and traits differ, spurious associations may arise due to confounding by population substructure (Pritchard et al., 2000; Risch et al., 2002; Tang et al., 2005). Due to this heterogeneity, genetic admixture analysis offers a unique opportunity for studying the role of genetic factors within a single, admixed population, independent of social factors, and comorbidities. Ancestry informative markers, AIMs, are genetic loci showing alleles with large frequency differences between populations that can be used to estimate bio-geographical ancestry at the level of the population and individual. Ancestry estimates at both the subgroup and individual level can be directly instructive regarding the genetics of the phenotypes that differ qualitatively or in frequency between populations (Shriver et al., 2003). Specifically, an association between genetic ancestry and a disease phenotype within an admixed group such as AAs may be an indicator of genetic factors underlying differential expression among racial groups (Peralta et al., 2010).

Adiponectin, an adipose-specific protein, is generally negatively associated with adiposity, insulin sensitivity, and diabetes (Cnop et al., 2003; Duncan et al., 2004; Cote et al., 2005; Li et al., 2009). Nevertheless, it has been shown that ethnicity modifies the relationships of adiponectin and insulin resistance with obesity. For example, a recent study showed that BMI, insulin, and insulin resistance assessed through the homeostasis model assessment (HOMA) correlated significantly with adiponectin levels only in Caucasian women (Hulver et al., 2004). In another study, normal weight African women showed marginally lower adiponectin levels than their Caucasian counterparts (Schutte et al., 2007). Moreover, no differences in adiponectin were shown for overweight and obese African and Caucasian women (Schutte et al., 2007). These results indicate that what applies to other ethnic populations might not apply to the AA population, and that the association between adiponectin and insulin sensitivity needs to be clarified in the AA population.

The studies indicate that polymorphisms at the adiponectin locus are predictors of circulating adiponectin levels, insulin sensitivity, and atherosclerosis, highlighting the pivotal role of this adipokine in the modulation of metabolism and atherogenesis (Menzaghi et al., 2007). Several genetic loci are determinants of adiponectin levels, genetic determinants that taken together influence risk of type 2 diabetes and insulin resistance (Dastani et al., 2012). Variability at the adiponectin locus associated with obesity and other features of the insulin resistance syndrome has been studies intensively in the last years (Comuzzie et al., 2001; Menzaghi et al., 2007), but a limited number of studies have examined the role of heredity in the regulation of adiponectin among individuals of African heritage (Miljkovic-Gacic et al., 2007). Estimates of adiponectin heritability (adjusted for age, gender, and BMI) in two populations of African descent were 0.45 and 0.70 for the African American and Nigerian families, respectively, indicating a relatively high genetic determinant for adiponectin levels (Hicks et al., 2007). As our recent Mendelian randomization collaborative study indicated, by using an ADIPOQ genetic summary risk score, no evidence of an association between adiponectin-lowering alleles and insulin sensitivity or type 2 diabetes appears present (Yaghootkar et al., 2013). As our results suggest, measures of global individual ancestry obtained with relevant frequency genetic markers might be helpful for controlling spurious associations-relevant to this important adipokine-detected in population studies due to population stratification and admixture.

Our study showing a direct association between global proportion of European ancestry and adiponectin suggests that genetic factors are a significant source of inter-individual differences in circulating adiponectin among AAs. Moreover, as the effect 
modification (interaction) of obesity and insulin resistance status of the relation of adiponectin levels with PEA indicates, a complex interplay among specific genetic loci and non-genetic factors, which may both be associated with the overall admixture, might lead to the observed ethnic differences in cardio-metabolic risk. As we have shown (Bidulescu et al., 2013b), in JHS there are negative correlations between adiponectin and many of the CVD risk factors yet the women, who have higher adiponectin levels than men, also have higher CVD risk factors. If adiponectin acts as a compensatory mechanism to counteract these risk factors warrants additional studies.

An alternative mechanistic explanation for our findings may be that the association between ancestry and adiponectin is due to some non-genetic confounders, which were not characterized in our investigation. Although we adjusted for many of the known correlates of adiponectin levels, it is possible that other differences in medical treatment or additional socioeconomic and psychosocial factors for which we do not have information may underlie some of the observed associations.

\section{STRENGTHS AND LIMITATIONS}

Our study sample is localized to one geographical area and one ethnic group, so generalizability inherently cannot be inferred. As an "aggregate" measure, the global individual proportion of European ancestry might not capture the genetic influences of specific genetic regions. These caveats are compensated by the fact that we used a large number of AIMs, and were able to control for a large number of potential confounders in a large sample of AAs.

\section{CONCLUSION}

Our study results point to the interaction of genetic and metabolic factors as variables associated with the lower levels of adiponectin in AAs. It warrants thus further exploration of the role ancestry plays in the complex relationship of adiponectin with cardio-metabolic risk factors.

\section{ACKNOWLEDGMENTS}

The authors thank the other investigators, the staff, and the participants of the JHS study for their valuable contributions. A full list of participating JHS investigators and institutions can be found at http://jhs.jsums.edu/jhsinfo/Directory/ tabid/55/Default.aspx. The Jackson Heart Study is supported and conducted in collaboration with Jackson State University (N01-HC-95170), University of Mississippi Medical Center (N01-HC-95171), and Tougaloo College (N01-HC-95172) NIH contracts from the National Heart, Lung, and Blood Institute (NHLBI) and the National Center on Minority Health and Health Disparities (NCMHD) with additional support from NHLBI contract HL076784, the National Institute of Aging (AG028321) and the National Institute on Biomedical Imaging and Bioengineering. This study was partially supported by PHS Award UL1 RR025008 from the National Institutes of Health, National Center for Research Resources to Dr. Bidulescu who was also supported by the NIH grant UH1 HL073461 provided by the National Heart, Lung and Blood Institute. The interpretation and opinions expressed in this article are those of the authors and do not necessarily reflect those of NIH or other federal agencies. The results described in this article were presented in part during the American Heart Association Scientific Conference, March 2012 in San Diego, California.

\section{REFERENCES}

Allison, M. A., Peralta, C. A., Wassel, C. L., Aboyans, V., Arnett, D. K., Cushman, M., et al. (2010). Genetic ancestry and lower extremity peripheral artery disease in the multi-ethnic study of atherosclerosis. Vasc. Med. 15, 351-359. doi: $10.1177 / 1358863 X 10375586$

Bidulescu, A., Liu, J., Chen, Z., Hickson, D. A., Musani, S. K., Samdarshi, T. E., et al. (2013a). Associations of adiponectin and leptin with incident coronary heart disease and ischemic stroke in African Americans: the Jackson Heart Study. Front. Public Health 1:16. doi: 10.3389/fpubh.2013.00016

Bidulescu, A., Liu, J., Hickson, D. A., Hairston, K. G., Fox, E. R., Arnett, D. K., et al. (2013b). Gender differences in the association of visceral and subcutaneous adiposity with adiponectin in African Americans: the Jackson Heart Study. BMC Cardiovasc. Disord. 13:9. doi: 10.1186/1471-2261-13-9

Bidulescu, A., Liu, J., Musani, S. K., Fox, E. R., Samdarshi, T. E., Sarpong, D. F., et al. (2011). Association of adiponectin with left ventricular mass in blacks: the Jackson Heart Study. Circ. Heart Fail. 4, 747-753. doi: 10.1161/CIRCHEARTFAILURE.110.959742

Bonilla, C., Parra, E. J., Pfaff, C. L., Dios, S., Marshall, J. A., Hamman, R. F., et al. (2004). Admixture in the Hispanics of the San Luis Valley, Colorado, and its implications for complex trait gene mapping. Ann. Hum. Genet. 68, 139-153. doi: 10.1046/j.1529-8817.2003.00084.x

Burchard, E. G., Ziv, E., Coyle, N., Gomez, S. L., Tang, H., Karter, A. J., et al. (2003). The importance of race and ethnic background in biomedical research and clinical practice. N. Engl. J. Med. 348, 1170-1175. doi: 10.1056/NEJMsb025007

Campbell, C. D., Ogburn, E. L., Lunetta, K. L., Lyon, H. N., Freedman, M. L., Groop, L. C., et al. (2005). Demonstrating stratification in a European American population. Nat. Genet. 37, 868-872. doi: 10.1038/ng1607

Carpenter, M. A., Crow, R., Steffes, M., Rock, W., Heilbraun, J., Evans, G., et al. (2004). Laboratory, reading center, and coordinating center data management methods in the Jackson Heart Study. Am. J. Med. Sci. 328, 131-144. doi: 10.1097/00000441-200409000-00001

Chakraborty, R., Ferrell, R. E., Stern, M. P., Haffner, S. M., Hazuda, H. P., and Rosenthal, M. (1986). Relationship of prevalence of non-insulin-dependent diabetes mellitus to Amerindian admixture in the Mexican Americans of San Antonio, Texas. Genet. Epidemiol. 3, 435-454. doi: 10.1002/gepi.1370030608

Chakraborty, R., and Weiss, K. M. (1986). Frequencies of complex diseases in hybrid populations. Am. J. Phys. Anthropol. 70, 489-503. doi: 10.1002/ajpa.1330700408

Choi, K. M., Lee, J., Lee, K. W., Seo, J. A., Oh, J. H., Kim, S. G., et al. (2004). The associations between plasma adiponectin, ghrelin levels and cardiovascular risk factors. Eur. J. Endocrinol. 150, 715-718. doi: 10.1530/eje.0.1500715

Cnop, M., Havel, P. J., Utzschneider, K. M., Carr, D. B., Sinha, M. K., Boyko, E. J., et al. (2003). Relationship of adiponectin to body fat distribution, insulin sensitivity and plasma lipoproteins: evidence for independent roles of age and sex. Diabetologia 46, 459-469. doi: 10.1007/s00125-003-1074-z

Comuzzie, A. G., Funahashi, T., Sonnenberg, G., Martin, L. J., Jacob, H. J., Black, A. E., et al. (2001). The genetic basis of plasma variation in adiponectin, a global endophenotype for obesity and the metabolic syndrome. J. Clin. Endocrinol. Metab. 86, 4321-4325. doi: 10.1210/jcem.86.9.7878

Cote, M., Mauriege, P., Bergeron, J., Almeras, N., Tremblay, A., Lemieux, I., et al. (2005). Adiponectinemia in visceral obesity: impact on glucose tolerance and plasma lipoprotein and lipid levels in men. J. Clin. Endocrinol. Metab. 90, 1434-1439. doi: 10.1210/jc.2004-1711

Dastani, Z., Hivert, M. F., Timpson, N., Perry, J. R., Yuan, X., Scott, R. A., et al. (2012). Novel loci for adiponectin levels and their influence on type 2 diabetes and metabolic traits: a multi-ethnic meta-analysis of 45,891 individuals. PLoS Genet. 8:e1002607. doi: 10.1371/journal.pgen.1002607

Dubbert, P. M., Carithers, T., Ainsworth, B. E., Taylor, H. A. Jr., Wilson, G., and Wyatt, S. B. (2005). Physical activity assessment methods in the Jackson Heart Study. Ethn. Dis. 15(4 Suppl 6), S6-56-S6-61.

Duncan, B. B., Schmidt, M. I., Pankow, J. S., Bang, H., Couper, D., Ballantyne, C. M., et al. (2004). Adiponectin and the development of type 2 diabetes: the atherosclerosis risk in communities study. Diabetes 53, 2473-2478. doi: 10.2337/diabetes.53.9.2473 
Gayoso-Diz, P., Otero-Gonzalez, A., Rodriguez-Alvarez, M. X., Gude, F., Garcia, F., De Francisco, A., et al. (2013). Quintela, Insulin resistance (HOMA-IR) cutoff values and the metabolic syndrome in a general adult population: effect of gender and age: EPIRCE cross-sectional study. BMC Endocr. Disord. 13:47. doi: 10.1186/1472-6823-13-47

Go, A. S., Mozaffarian, D., Roger, V. L., Benjamin, E. J., Berry, J. D., Blaha, M. J., et al. (2014). Heart disease and stroke statistics-2014 update: a report from the American heart association. Circulation 129, e28-e292. doi: 10.1161/01.cir.0000441139.02102.80

Hanis, C. L., Chakraborty, R., Ferrell, R. E., and Schull, W. J. (1986). Individual admixture estimates: disease associations and individual risk of diabetes and gallbladder disease among Mexican-Americans in Starr County, Texas. Am. J. Phys. Anthropol. 70, 433-441. doi: 10.1002/ajpa.1330700404

Hicks, C., Zhu, X., Luke, A., Kan, D., Adeyemo, A., Wu, X., et al. (2007). A genomewide scan of loci linked to serum adiponectin in two populations of African descent. Obesity (Silver Spring) 15, 1207-1214. doi: 10.1038/oby.2007.142

Hulver, M. W., Saleh, O., MacDonald, K. G., Pories, W. J., and Barakat, H. A. (2004). Ethnic differences in adiponectin levels. Metabolism 53, 1-3. doi: 10.1016/j.metabol.2003.07.002

Li, S., Shin, H. J., Ding, E. L., and van Dam, R. M. (2009). Adiponectin levels and risk of type 2 diabetes: a systematic review and meta-analysis. JAMA 302, 179-188. doi: 10.1001/jama.2009.976

Long, J. C. (1991). The genetic structure of admixed populations. Genetics 127, 417-428.

Matthews, D. R., Hosker, J. P., Rudenski, A. S., Naylor, B. A., Treacher, D. F., and Turner, R. C. (1985). Homeostasis model assessment: insulin resistance and beta-cell function from fasting plasma glucose and insulin concentrations in man. Diabetologia 28, 412-419. doi: 10.1007/BF00280883

Menzaghi, C., Trischitta, V., and Doria, A. (2007). Genetic influences of adiponectin on insulin resistance, type 2 diabetes, and cardiovascular disease. Diabetes 56, 1198-1209. doi: 10.2337/db06-0506

Miljkovic-Gacic, I., Wang, X., Kammerer, C. M., Bunker, C. H., Wheeler, V. W., Patrick, A. L., et al. (2007). Genetic determination of adiponectin and its relationship with body fat topography in multigenerational families of African heritage. Metabolism 56, 234-238. doi: 10.1016/j.metabol.2006.09.019

Musunuru, K., Lettre, G., Young, T., Farlow, D. N., Pirruccello, J. P., Ejebe, K. G., et al. (2010). Candidate gene association resource (CARe): design, methods, and proof of concept. Circ. Cardiovasc. Genet. 3, 267-275. doi: 10.1161/CIRCGENETICS.109.882696

Ouchi, N., Shibata, R., and Walsh, K. (2006). Cardioprotection by adiponectin. Trends Cardiovasc. Med. 16, 14114-14116. doi: 10.1016/j.tcm.2006.03.001

Parra, E. J., Kittles, R. A., Argyropoulos, G., Pfaff, C. L., Hiester, K., Bonilla, C., et al. (2001). Ancestral proportions and admixture dynamics in geographically defined African Americans living in South Carolina. Am. J. Phys. Anthropol. 114, 18-29. doi: 10.1002/1096-8644(200101)114:1 < 18::AID-AJPA1002>3.0.CO;2-2

Parra, E. J., Marcini, A., Akey, J., Martinson, J., Batzer, M. A., Cooper, R., et al. (1998). Estimating African American admixture proportions by use of population-specific alleles. Am. J. Hum. Genet. 63, 1839-1851. doi: 10.1086/302148

Patel, D. A., Srinivasan, S. R., Xu, J. H., Chen, W., and Berenson, G. S. (2006). Adiponectin and its correlates of cardiovascular risk in young adults: the Bogalusa heart study. Metabolism 55, 1551-1557. doi: 10.1016/j.metabol.2006.06.028

Peralta, C. A., Risch, N., Lin, F., Shlipak, M. G., Reiner, A., Ziv, E., et al. (2010). The Association of African Ancestry and elevated creatinine in the Coronary Artery Risk Development in Young Adults (CARDIA) Study. Am. J. Nephrol. 31, 202-208. doi: 10.1159/000268955

Pritchard, J. K., Stephens, M., Rosenberg, N. A., and Donnelly, P. (2000). Association mapping in structured populations. Am. J. Hum. Genet. 67, 170-181. doi: 10.1086/302959

Risch, N., Burchard, E., Ziv, E., and Tang, H. (2002). Categorization of humans in biomedical research: genes, race and disease. Genome Biol. 3, comment 2007. doi: 10.1186/gb-2002-3-7-comment2007

Schutte, A. E., Huisman, H. W., Schutte, R., Malan, L., van Rooyen, J. M., Malan, N. T. et al. (2007). Differences and similarities regarding adiponectin investi- gated in African and Caucasian women. Eur. J. Endocrinol. 157, 181-188. doi: 10.1530/EJE-07-0044

Shriver, M. D., Parra, E. J., Dios, S., Bonilla, C., Norton, H., Jovel, C., et al. (2003). Skin pigmentation, biogeographical ancestry and admixture mapping. Hum. Genet. 112, 387-399. doi: 10.1007/s00439-002-0896-y

Smith, M. W., Patterson, N., Lautenberger, J. A., Truelove, A. L., McDonald, G. J., Waliszewska, A., et al. (2004). A high-density admixture map for disease gene discovery in african americans. Am. J. Hum. Genet. 74, 1001-1013. doi: $10.1086 / 420856$

Tang, H., Quertermous, T., Rodriguez, B., Kardia, S. L., Zhu, X., Brown, A., et al. (2005). Genetic structure, self-identified race/ethnicity, and confounding in case-control association studies. Am. J. Hum. Genet. 76, 268-275. doi: $10.1086 / 427888$

Taylor, H. A. Jr. (2005). The Jackson Heart Study: an overview. Ethn. Dis. 15(4 Suppl 6), S6-1-S6-3.

Tsai, H. J., Choudhry, S., Naqvi, M., Rodriguez-Cintron, W., Burchard, E. G., and Ziv, E. (2005). Comparison of three methods to estimate genetic ancestry and control for stratification in genetic association studies among admixed populations. Hum. Genet. 118, 424-433. doi: 10.1007/s00439-005-0067-z

Vettor, R., Milan, G., Rossato, M., and Federspil, G. (2005). Review article: adipocytokines and insulin resistance. Aliment. Pharmacol. Ther. 22, 3-10. doi: 10.1111/j.1365-2036.2005.02587.x

Wannamethee, S. G., Lowe, G. D., Rumley, A., Cherry, L., Whincup, P. H., and Sattar, N. (2007). Adipokines and risk of type 2 diabetes in older men. Diabetes Care 30, 1200-1205. doi: 10.2337/dc06-2416

Wassel, C. L., Pankow, J. S., Peralta, C. A., Choudhry, S., Seldin, M. F., and Arnett, D. K. (2009). Genetic ancestry is associated with subclinical cardiovascular disease in African-Americans and Hispanics from the multiethnic study of atherosclerosis. Circ. Cardiovasc. Genet. 2, 629-636. doi: 10.1161/CIRCGENETICS.109.876243

Wassel Fyr, C. L., Kanaya, A. M., Cummings, S. R., Reich, D., Hsueh, W. C., Reiner, A. P., et al. (2007). Genetic admixture, adipocytokines, and adiposity in Black Americans: the health, aging, and body composition study. Hum. Genet. 121, 615-624. doi: 10.1007/s00439-007-0353-z

Wyatt, S. B., Diekelmann, N., Henderson, F., Andrew, M. E., Billingsley, G., Felder, S. H., et al. (2003). A community-driven model of research participation: the Jackson Heart Study participant recruitment and retention study. Ethn. Dis. 13, 438-455.

$\mathrm{Xu}, \mathrm{S}$., and Jin, L. (2008). A genome-wide analysis of admixture in Uyghurs and a high-density admixture map for disease-gene discovery. Am. J. Hum. Genet. 83, 322-336. doi: 10.1016/j.ajhg.2008.08.001

Yaghootkar, H., Lamina, C., Scott, R. A., Dastani, Z., Hivert, M. F., Warren, L. L., et al. (2013). Mendelian randomization studies do not support a causal role for reduced circulating adiponectin levels in insulin resistance and type 2 diabetes. Diabetes 62, 3589-3598. doi: 10.2337/db13-0128

Conflict of Interest Statement: The authors declare that the research was conducted in the absence of any commercial or financial relationships that could be construed as a potential conflict of interest.

Received: 26 November 2013; accepted: 21 January 2014; published online: 10 February 2014.

Citation: Bidulescu A, Choudhry S, Musani SK, Buxbaum SG, Liu J, Rotimi CN, Wilson JG, Taylor HA and Gibbons GH (2014) Associations of adiponectin with individual European ancestry in African Americans: the Jackson Heart Study. Front. Genet. 5:22. doi: 10.3389/fgene.2014.00022

This article was submitted to Applied Genetic Epidemiology, a section of the journal Frontiers in Genetics.

Copyright (c) 2014 Bidulescu, Choudhry, Musani, Buxbaum, Liu, Rotimi, Wilson, Taylor and Gibbons. This is an open-access article distributed under the terms of the Creative Commons Attribution License (CC BY). The use, distribution or reproduction in other forums is permitted, provided the original author(s) or licensor are credited and that the original publication in this journal is cited, in accordance with accepted academic practice. No use, distribution or reproduction is permitted which does not comply with these terms. 